\section{Computational Modeling of Damage Growth in Composite Laminates}

\author{
Shiladitya Basu, Anthony M. Waas \\ and Damodar R. Ambur ${ }^{1}$
}

\author{
February 5th,2003 \\ Composite Structures Laboratory \\ Department of Aerospace Engineering \\ University of Michigan, Ann Arbor, MI, 48109-2140
}

\section{Abstract}

A progressive damage growth model, based on a system of orthotropic nonlinear elastic relations and a set of internal state variables, previously developed by the authors and designated as EST (extended Schapery theory, after Schapery) for laminated fiber reinfoced composites is applied to model the compression response of a stiffened builtup laminated structure for which experimental results are available. The mechanics of damage initiation and growth in a single lamina is modeled in a 2D plane stress setting. Damage evolution is controlled via the solution of a thermodynamically consistent set of equations at each load step in conjunction with a criterion for compressive fiber kinking in the zero plies. The commercially available finite element (FE) analysis package ABAQUS was used to perform the numerical simulations. A user defined material (UMAT) was written for the EST material

\footnotetext{
${ }^{1}$ Graduate Research Assistant, Professor of Aerospace Engineering, University of Michigan, and, Head, Mechanics and Durability Branch, NASA Langley Research Center, Hampton, VA, 23681 respectively. Copyright (C)2003 by Anthony M. Waas. Published by the AIAA, with permission.
}

constitutive behavior which was used with the existing finite element library in ABAQUS. Good agreement between experimental results (for load-panel end shortening, panel surface strains, out-of-plane buckling displacements) and the FE results is reported for the case of a notched axially compressed stiffened panel.

\section{Introduction}

Development of computational methodologies for the prediction of progressive damage growth in continuous fiber composite laminates is presently an active area of research. Available predictive methods use pre-defined strength criteria at the lamina level. Based on critical values for tensile, compressive and shear 'strengths', these methods compute damage indices that are expressed in functional form in terms of the current stress state. When any of these indices exceeds a critical value, the material is said to have failed [1]. Beyond initial failure, a consistent and rigorous methodology to account for progressive material deterioration has not been investigated thoroughly. An exception to this is the study by Schapery and Sicking [2]. Schapery and Sicking[2] carried out lamina level tests, and validated the test results by developing a thermodynamically based progressive damage formation and growth model. They assumed that the fiber direction response is essentially linear (slight elastic nonlinearity in the fiber direction was accounted for), but, damage (microcracking and transverse cracking) formation affects the response in the transverse direction. Consequently, internal state variables that are related to the damage mechanisms in the transverse direction were identified and evolution laws that specify the growth of damage and hence its influence on the transverse direction response were prescribed. In the present paper, Schapery's theory (ST) is extended to account for fiber direction damage (both, in tension and compression) by identifying an additional internal state variable associated with the fiber direction response. This extension, referred to as the extended Schapery theory (EST) has been successfully applied to study the damage growth and evolution in notched unidirectional plates by the authors[3]. The present paper is concerned with the application 
AIAA-2003-1598

of EST to study damage initiation and growth in a axially compressed stiffened stitched panel which has been investigated by [4].

In unidirectional lamina, transverse direction damage accumulation results in progressively decreasing but smooth variations in instantaneous tangent moduli. In contrast, damage accumulation in the fiber direction leads to non-smooth abrupt changes in the corresponding moduli. These changes must be properly captured if progressive damage growth in fiber reinforced composite laminates is to be modeled accurately.

Coupon level tests in the fiber direction, transverse to the fiber direction and off axis tests are commonly used to obtain material behavior at the lamina level. These tests supply a complete response (stress-strain) curve (usually non-linear) with valuable information beyond the proportional limit. The present theory (EST) is based on input that uses only these measured and available test data in conjunction with the laminate stacking sequence and geometry of the problem configuration. Thus, the EST introduced here uses readily available coupon level experimental input. In using the methodology introduced to predict damage growth in a general laminate, it is assumed that the laminate is built-up as a stack of lamina. Each lamina is modeled as a nonlinear elastic, homogenised, orthotropic layer that can undergo damage. Nonlinear elastic behavior is depicted via three polynomial functions of strain. Two of these functions describe the ratio of secant moduli in fiber and transeverse directions with the initial values of the respective moduli, without damage. The third function describes the behavior of poisson's ratio. Damage is incorporated via three internal state variables (ISV). These ISV's represent the 'thermodynamic forces' to change the state of a material system. Evolution laws of these ISV's incorporate work considerations and thermodynamic requirements, such as, increase in entropy.

\section{Theoretical Development}

\subsection{Schapery Theory - Damage Mod- eling Through Internal State Variables}

Even though elements of EST are described in [5], for completeness we provide the reader with the necessary background to use EST. Schapery [6] developed nonlinear elastic consitutive relations using a work potential approach which accounted for the effect of microdamage in composite lamina. Individual internal field variables $\left(S_{i}\right)$ were used to characterize and monitor the progress of each of the damage modes present for a laminated composite. These ISVs represent the nonrecoverable portion of the total energy (Figure 1). Evolution laws for the ISVs can be obtained by comparing the "thermodynamic force' required for progression of a damage mode in the undamaged material system (equation 1) with the energy available for damage growth at every stress state (equation 2).

$$
\begin{gathered}
f_{i}=\frac{\partial W_{s}}{\partial S_{i}} \\
f_{i} \equiv-\frac{\partial W}{\partial S_{i}}
\end{gathered}
$$

where, $W_{s}$ is the total nonrecoverable portion of total energy and $W$ is the total energy of the system at a particular stress state. Each ISV, would also have to satisfy the non-positive rate of entropy change (equation 3) as 'healing' is not allowed for the class of materials considered here.

$$
\sum \dot{S}_{i} \geq 0
$$

In ref [2], two internal state variables $\left(S_{r}\right.$ and $S_{c}$ ) were modeled and necessary modeling charteristics were developed for a carbon-fiber reinforced composite laminate system. Fiber direction elastic modulus $\left(E_{11}\right)$ and principal poisson's ratio $\left(\nu_{12}\right)$ were considered to be unaffected by these ISVs. Only the transverse modulus $\left(E_{22}\right)$ and shear modulus $\left(G_{12}\right)$ change with the progression of damage as considered in [6]. 


\subsection{Fiber Direction Damage - Ex- tended Schapery Theory (EST)}

The theory presented in [6] is for a continuous evolution of the stress-strain relations in the damaging material. It needs to be extended to to incorporate post-kinking response (compression) or post-fracture response (tension), where the instantaneous material moduli undergo abrupt changes. The salient features of the fiber direction response of a unidirectional lamina are shown in figure 2. These features have been observed by a number of reseachers and the reader is referred to ([7]- [10]), for experimental evidence.

A new $I S V$ associated with fiber direction damage is introduced in [5] to incorporate response beyond kinking (compression) and fiber fracture (tension). It has been observed in laboratory tests that a material behaves differently for tensile and compressive loading along the fiber direction. While, local fiber microbuckling/kinking due to the presence of local imperfections is the main mode of failure in compression, tensile failure is due to fiber fracture, appearing as cracks perpendicular to the loading direction.

$$
\begin{gathered}
W_{s}=S_{w}+S+S_{c} \\
W=Q_{11} I_{11}+Q_{22} I_{22}+\nu_{12} Q_{22} I_{1} I_{2}+\frac{G_{12} \gamma_{12}^{2}}{2}
\end{gathered}
$$

where, $I_{i j}$ are functions of strain (for further discussion about $I_{i j}$ s, refer to [2]).

$$
\begin{aligned}
I_{11} & \equiv \int_{0}^{\bar{\epsilon}_{1}} \epsilon_{1} d \epsilon_{1} \\
I_{22} & \equiv \int_{0}^{\bar{\epsilon}_{2}} \epsilon_{2} f_{2} d \epsilon_{2} \\
I_{1} & \equiv \int_{0}^{\bar{\epsilon}_{1}} f_{12} d \epsilon_{1} \\
I_{2} & \equiv \int_{0}^{\bar{\epsilon}_{2}} f_{2} d \epsilon_{2}
\end{aligned}
$$

The tensile response shows a clear loss of stiffness at failure and the residual stiffness and strength diminsh to zero immediately after failure. The compressive response shows the presence of residual strength after failure giving an impression of transforming into a different new material. That is, if the material is unloaded immediately beyond failure and re-loaded, then it appears to carry load, albeit, at a reduced stiffness. Furthermore, the maximum strength of the post-kinked material appears to be bounded by a plateau stress. We note that Rajagopal et al, [11] have established a general thermodynamic framework to address material microstructure evolution in a finite strain setting, with application to a variety of phenomena ranging from scission in polymers to thermal degradation in rubbery solids.

The $I S V, S_{w}$ represents the inelastic behavior in the fiber direction due to compressive kinking or fiber fracture. Hence, $S_{w}$ is not present in the initial elastic regime. It can be computed from the material stress-strain curve under compressive loading in the fiber direction (figure 3). Once kinking (compression) or fiber fracture (tension) occurs, $S_{w}$ takes a finite jump. The finite jump in $S_{w}$ in compression is termed the kinking toughness and is a material property that is unique to a fiber reinforced lamina. A set of laboratory scale experiments can be carried out to systematically measure this quantity. $S_{w}$ is related to the fiber direction secant modulus, $E_{11}$ via relations similar to the moduli in other directions.

$$
E_{11}=E_{11_{0}} h\left(S_{w}\right)
$$

Using equations ( 1,2 and 4 ) we can obtain the $S_{w}$ evolution law at every state of strain as,

$$
I_{11} \frac{\partial E_{11}}{\partial S_{w}}=-1
$$

\section{Application of EST - Stiff- ened Panel with a Notch}

Experiments on a stiffened, stitched, thick composite laminated panel were conducted at NASA Langley Research Center ([12]). Finite element based numerical simulations of the experiments were performed using Hashins' criteria ([13]) and Chang 
and Lessards' ([14]) property degradation model, as reported in [4]. The numerical simulations were carried out using the finite element package ABAQUS. In [4] results are presented for the compression response of three centrally notched panels with different notch angles. In particular, numerical results were presented for the applied load-end shortening response, (the $P-\Delta$ curve), out of plane deflection at the center of the panel as a function of $\Delta$ and, surface strain values at selected locations. The locations coincided with the sites where strain gage readings were recorded during the panel compression tests. The agreement between experiment and prediction was good, however, several adjustments to the strength values adopted in the property degradation model had to be made in order to incorporate the effect of stitching on altering the fiber direction compressive strength value, ([4]).

\subsection{Geometry}

The EST material model introduced in the previous section was numerically implemented to generate results that can be compraed against the experimental results reported in ([4])for the compression response of a panel with a notch angle of $\alpha=15^{0}$ (P-15). Figure 4 shows the problem geometry with salient features. Finite element mesh of the problem is shown in Figure 5. Three stiffeners are stitched to one side of the panel skin with Kevlar stitches. A central notch is cut at the center of the panel through the central stiffener. Displacement boundary conditions at the ends of the panel were used to apply far field loading. Ends of the panel were subjected to fully clamped boundary conditions. The sides of the panel were assumed to be simply supported (out-of-plane) and allowed to freely displace in the in-plane directions. This simulates the anti-buckling restraints used in the experiments reported in [4].

\subsection{Material Model}

In the commercially available FE code ABAQUS, it is possible to introduce user defined material behavior through user written subroutines. To that end, the user needs to provide the incremental stress-strain relationships or the material ja- cobian $(\mathrm{J})$ and update the stress and/or any internal state variables to their respective values at the end of each increment. The ABAQUS finite element solver calls this user subroutine at every material point, where the user defined material option is included ([15]).

The material data for the present study were obtained from [4] and are given in Table 1. The material is modeled as a homogenised orthotropic nonlinear elastic material obeying EST. The panel, P-15, consists of nine stacks of material stitched together by stitches. Each stack has seven loosely tied plies having a lay-up of $[45 /-45 / 0 / \overline{9} 0]_{s}$. The 0 -deg orientation plies in the panel skin were made of $I M 7 C / 3501-6$. All the other plies in the skin were made of $A S 4 / 3501-6$ material system. For stiffeners, all plies are made of $A S 4 / 3501-6$ material system. Relevant geometric data are tabulated in Table 2. The material parameters needed to model the panel materials as EST materials were obtained from ref [2]. Representative data for the $0-$ deg layer are shown in Table 3. Detail discussion of obtaining these parameters are given in [5]. No attempt was made to accomodate local effects of the stitches, due to lack of detailed knowledge pertaining to the effects of stitches in altering the zero ply properties.

\subsection{Element and Analysis Type}

The existing element library of ABAQUS version 6.3 was used to model the panel for the analyses. Reduced integration bi-linear four noded shell elements, S4R5 and three noded shell elements, S3R were used. In ABAQUS, one single shell element can be used for through the thickness modeling of a complete laminate by specifying the individual layer thicknesses and orientations in the element section definition in the ABAQUS input file. Each layer would have $3-5$ integration points through the thickness for section property computation. This approach leads to extensive computational cost when applied to a 'thick' laminate containing more than 48 plies. A shell element superposition method as proposed and used in ([4], [16]) was used to model the laminated panel. Mesh parameters are tabulated in Table 4 . The out of plane bending effects were accomodated by using five integration points through the thickness. In order to verify the efficacy and 
accuracy of this modeling process, an example simulation of the response of a square laminated panel subjected to transverse pressure was simulated using the shell element superposition method and the standard approach (via classical lamination theory CLT). The superposition method was as accurate as the solution produced through CLT. Thus, in large scale problems, the superposed shell element approach is more efficient, as has also been reported in [4].

\section{Discussion of the Numerical Results}

Numerical data for the panel behavior under inplane compressive loading are presented in this section. The $P-\Delta$ curve compared against experiment is shown in Figure 6. The fiber direction stress-strain curve for this case is shown in Figure 7. Two curves are shown in this figure and labeled as case 1 and case 2 . In case 1 , the fiber direction kinking strain is 0.01 with a plateau stress value that is $50 \%$ of the maximum strength, whereas in case 2 , the kinking strain is set to 0.006 with a plateau stress that is $35 \%$ of the maximum strength. It is to be noted that the fiber direction strength and stiffness values are scaled appropriately to reflect the methodology used in the superposed shell element formulation. Other implementation details of the EST are reported in [3].

Experimental data for the panel reported in [4] shows a substantial loss of inplane stiffness around an applied end shortening of $0.09 \mathrm{inch}$. Also, there is sharp drop in resultant load around 0.125 inch of applied end shortening. The present EST based simulation captures the inplane response but is not able to reproduce the change in slope of the $P-\Delta$ response. This is attributed to the fact that EST assumes a kinking strain value for the zero plies that is fixed at 0.01 , for case 1 . Ideally, the zero ply kinking strain should be a function of the local state of the material, so that degradation of the transverse properties at a material point does influence the local value of the kinking strain. This local coupling between the kinking strain and the degraded transverse properties can be incorporated in the framework of the present EST formulation, however, this aspect is the subject of future work. It is to be noted that such coupling cannot be incorporated in a property degradation scheme such as the Chang and Lessard scheme [14]. A seperate analysis (case 2), was performed holding all other variables fixed but changing the value of kinking strain to $-6000 \mu$ strain and the plateau strength to $35 \%$ of the kinking strength. The results obtained with such a set of parameters are also indicated in Figure 6 . The reduction in kinking strain leads to a larger volume of kinked material and, as a result, the overall axial stiffness of the panel is degraded. This degradation is also influenced by the plateau strength. Ideally, the kinking toughness, [3] remains fixed, thus, this provides a way to change the salient features of the fiber direction stress-starin reposne while holding the kink toughness (area under the stress-strain curve) fixed. The degradation in the panel axial stiffness occurs gradually and also affects the out-of-plane displacement response. In the experimental results reported in [4], it is mentioned that the damage zone was observed to reach a particular location near the notch tip at approximately $-5500 \mu$ strain.

In figure 8 , the out of plane displacement $(w)$ is plotted against the applied end shortening. This figure is significant in the sense that, it clearly shows the effect of inplane stiffness loss on the out of plane motion of the panel. As can be seen, the center of the panel moves out from the unloaded configuration with applied compression. Then, as the end shortening reaches the point where a substantial change occurs in the inplane stiffness, the panel is not able to maintain its curvature anymore. With further application of load, the panel reverses its curvature at the center indicating local stress redistribution and stiffness loss. Again, EST with a kinking strain of 0.01 is not able to produce this feature in the out-ofplane displacement reponse. However, the lowering of the kinking strain criterion is able to capture the trend in panel behavior adequately. Exact reproduction of the experimental behavior is not achieved, perhaps due to the fact that other panel features such as stitch geometry and stitch placement have not been accounted for. A more refined model that includes these aspects as well as the effects of coupling between the fiber direction and the transverse 
direction may be able to produce better correlation. It is interesting to note that kinked elements undergo cycles of loading, unloading and further re-loading, thus capturing an essential feature of problems involving damage growth. That is, unlike the property degradation schemes reported in [14], a material point that undergoes kinking is still able to transmit stress in the post-kinked regime. In the scheme by [14], once a materail point is damaged, then it contributes no further to the stiffness of the panel.

Axial strain $\left(\epsilon_{11}\right)$ data are also simulated and plotted for five different locations, ahead of the notch tip. These locations were chosen such that they fall in the path of possible damage growth. These positions are shown in Figure 9. Strain data for these locations are plotted in Figure 11. A sudden increase in compressive strain indicates damage growth (or progression) through the observation point. It is seen that EST predictions (both cases) are able to model these responses fairly accurately.

\section{Conclusions}

A progressive damage growth model is proposed and applied to simulate the initiation and progression of damage in a stiffened stitched panel under inplane compression. The panel material is assumed to be governed by the extended schapery theory (EST). Details of EST have been reported before [5]. Comparison between experimental measurements and numerical predictions for the load vs. end shortening reponse, the out-of-plane displacement vs. end shortening reponse and the surface strain vs. end-shortening response showed that EST is capable of reproducing the salient features of the panel response. Refinements to the model that include coupling the fiber direction kinking strain with the local values of the degraded material properties is left for future work. The effect of kinking strain was investigated and it was shown that lowering of the fiber direction kinking strain to a value that conforms to the measured panel surface strains led to better agreement between experiment and prediction.
[1] Fu-Kuo Chang and K. Y. Chang. A Progressive Damage Model for Laminated Composites Containing Stress Concentrations. Journal of Composite Materials, 21:834-855, September 1987.

[2] R. A. Schapery and D. L. Sicking. On Nonlinear Constitutive Equations for Elastic and Viscoelastic Composites with Growing Damage. Mechanical Behavior of Materials, 47:4576, June 1995.

[3] Shiladitya Basu, Anthony M. Waas, and Damodar R. Ambur. Computational Modeling of Damage Growth in Composite Laminates. accepted for publication, 2003.

[4] Carlos G. Dávila, Damodar R. Ambur, and David M. McGowan. Analytical Prediction of Damage Growth in Notched Composite Panels Loaded in Compression. Journal of Aircraft, 37(5):898-905, September-October 2000.

[5] Shiladitya Basu, Anthony M. Waas, and Damodar R. Ambur. Computational Modeling of Damage Growth in Composite Laminates. $43^{\text {rd }}$ SDM Conference, AIAA, Denver, April 2002 .

[6] R. A. Schapery. Mechanical Characterization and Analysis of Inelastic Composite Laminates with Growing Damage. Mechanics of Composite Materials and Structures, AMD-100:1-9, June 1989.

[7] C. R. Schultheisz and A. M. Waas. Compressive Failure of Composites 1. Testing and Micromechanical Theories. PROG. AEROSP SCI, 32(1):1-42, 1996.

[8] A. M. Waas and C. R. Schultheisz. Compressive Failure of Composites 2. Experimental Studies. PROG. AEROSP SCI, 32(1):43-78, 1996.

[9] J. H. Ahn and Anthony M. Waas. Prediction of Compressive Failure in Laminated Composites at Room and Elevated Temperature. AIAA Journal, 40(2):346-358, February 2002.

[10] Chandra S. Yerramalli and Anthony M. Waas. Compressive Splitting Failure of Composites using Modified Shear Lag Theory. International Journal of Fracture, 115(1):27-40, May 2002. 
AIAA-2003-1598

[11] K. Rajagopal. On a Class of Elastodynamic Motions in a Neo-Hookean Elastic Solid. Internationa Journal of Non-Linear Mechanics, 33(3):397-405, 1998.

[12] D. C. Jegley and H. G. Bush. Structural test documentation and results for the mcdonnell douglas all-composite wing stub box. NASA TM-110204, April 1997.

[13] Z. Hashin. Failure Criteria for Unidirectional Fiber Composites. Journal of Applied Mechanics, 47:329-334, June 1980.

[14] Fu-Kuo Chang and Larry B. Lessard. Damage Tolerance of Laminated Composites Containing an Open Hole and Subjected to Compressive Loadings: Part i-Analysis. Journal of Composite Materials, 25:2-43, January 1991.

[15] ABAQUS User's Manual,Vol 3, Ver. 6.3-1, 2003.

[16] David M. McGowan, Carlos G. Dávila, and Damodar R. Ambur. Damage Progression in Buckle-Resistant Notched Composite Plates Loaded in Uniaxial Compression. 42 ${ }^{\text {nd }} S D M$ Conference, AIAA, Seattle, April 2001.

\section{Appendix}

\begin{tabular}{|c|c|c|c|c|}
\hline & \multicolumn{4}{|c|}{ Fiber system } \\
\hline & \multicolumn{3}{|c|}{ AS4 } & IM7C \\
\hline Orientations & 0 & 45 & 90 & 0 \\
\hline Moduli & & & & \\
$E_{L}, \mathrm{Msi}$ & 15.30 & 15.04 & 14.87 & 15.04 \\
$E_{T}, \mathrm{Msi}$ & 1.60 & 1.60 & 1.60 & 1.60 \\
$G_{L T}, \mathrm{Msi}$ & 0.8 & 0.8 & 0.8 & 0.8 \\
$\nu_{12}$ & 0.34 & 0.34 & 0.34 & 0.34 \\
\hline Thickness Fraction & 0.443 & 0.2175 & 0.122 & 0.443 \\
\hline
\end{tabular}

Table 1: Engineering Properties for the panel material, ref [4].

\begin{tabular}{|l|c|}
\hline Thicknesses & inch \\
\hline $0^{0}$ ply & 0.01285 \\
$45^{0}$ ply & 0.00633 \\
$90^{0}$ ply & 0.007018 \\
\hline Stack & 0.058 \\
Panel Skin & 0.535 \\
Stiffener Flange & 0.46 \\
\hline Length & 43.0 \\
Width & 19.0 \\
Inter Stiffener Distance & 7.00 \\
\hline
\end{tabular}

Table 2: Dimensions of the panel, ref [4].

\begin{tabular}{|c|c|c|c|c|}
\hline Exponent & $f_{1}$ & $f_{2}$ (comp) & $f_{2}$ (tens) & $f_{12}$ \\
\hline 0 & 1 & 1 & 1 & 1 \\
1 & 4.420 & 5.513 & -5.513 & -15.0881 \\
2 & -79.478 & -53.0287 & -81.9058 & -575.623 \\
\hline
\end{tabular}

Table 3: Parameters needed for EST modeling [2] (representative, $0^{0}$ layer).

\begin{tabular}{|c|c|}
\hline Mesh Parameters & Mesh for P-15 \\
\hline Nodes & 14576 \\
Degrees of freedom & 87456 \\
Elements & 57480 \\
Types of elements & $S 3, S 4$ \\
\hline
\end{tabular}

Table 4: Element and Node data for the mesh used 
AIAA-2003-1598

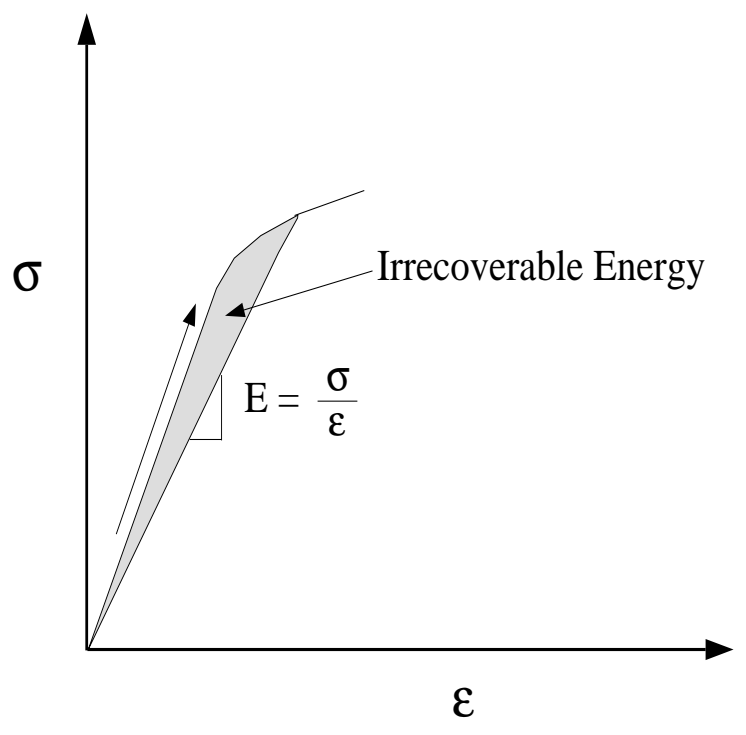

Figure 1: Irrecoverable energy computation from stress-strain response
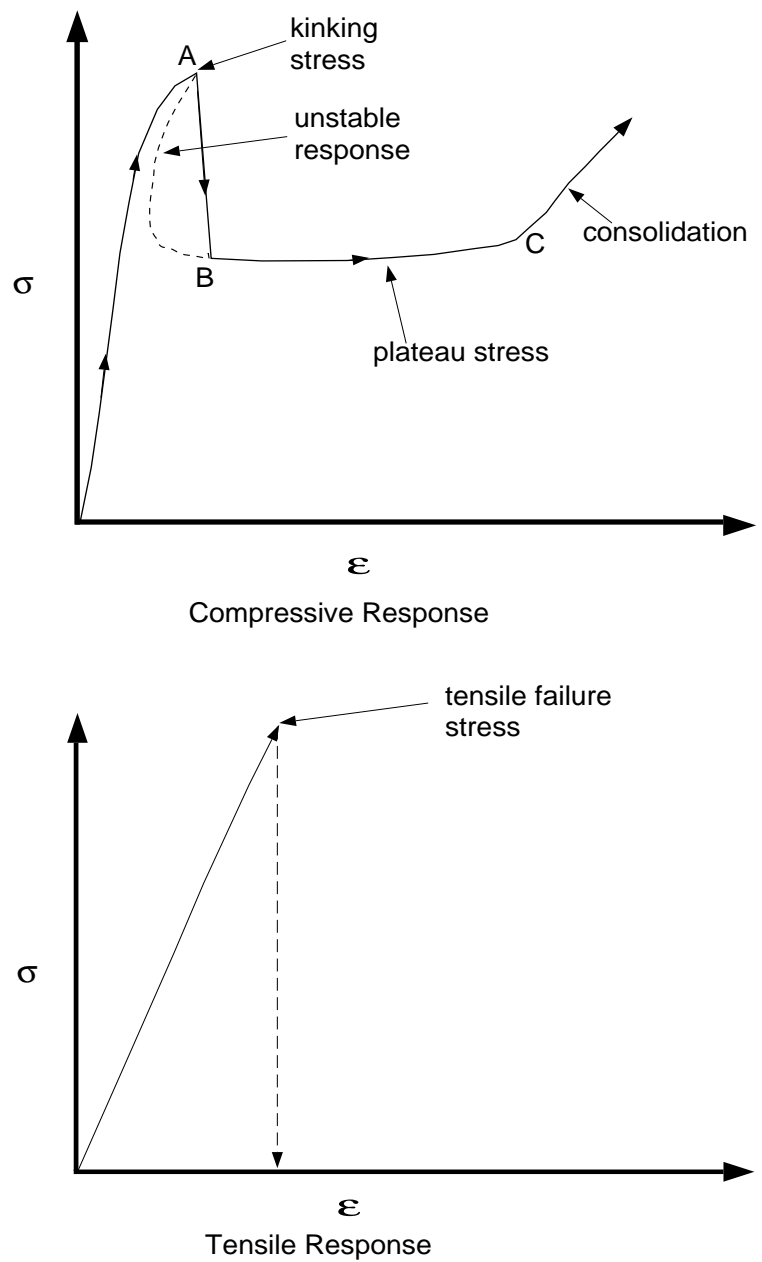

Figure 2: Typical material stress-strain behavior under uniaxial loading 


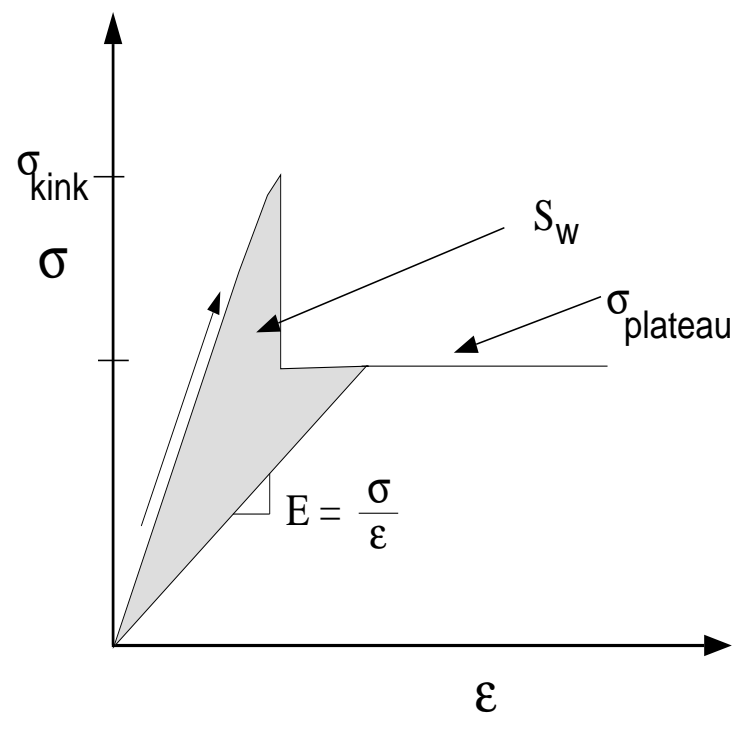

Figure 3: Determination of $S_{w}$ from stress-strain response

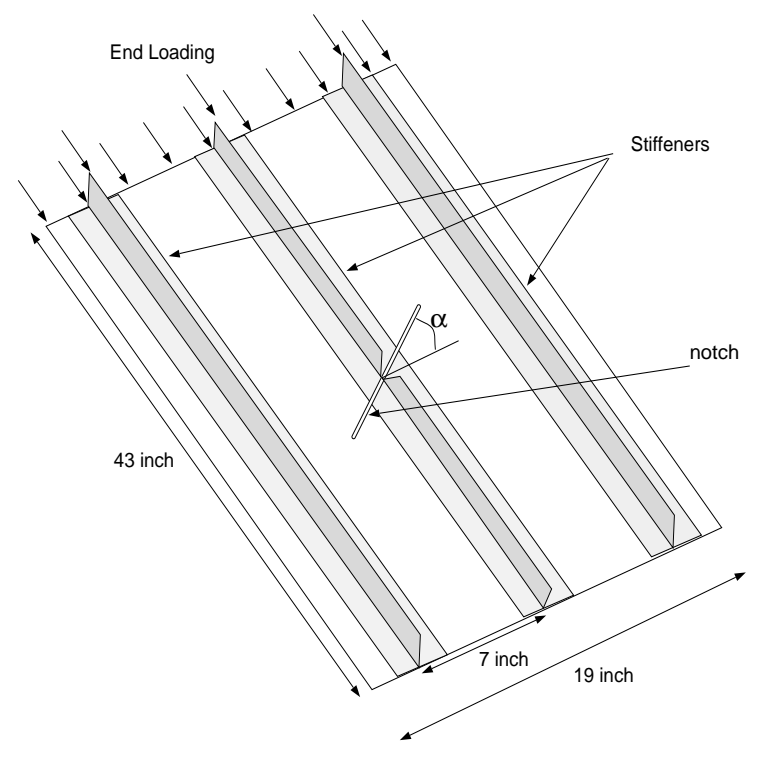

Figure 4: Geometry of the notched panel. Each panel has different thicknesses due to difference in stacking and compaction during manufacturing [4].
AIAA-2003-1598

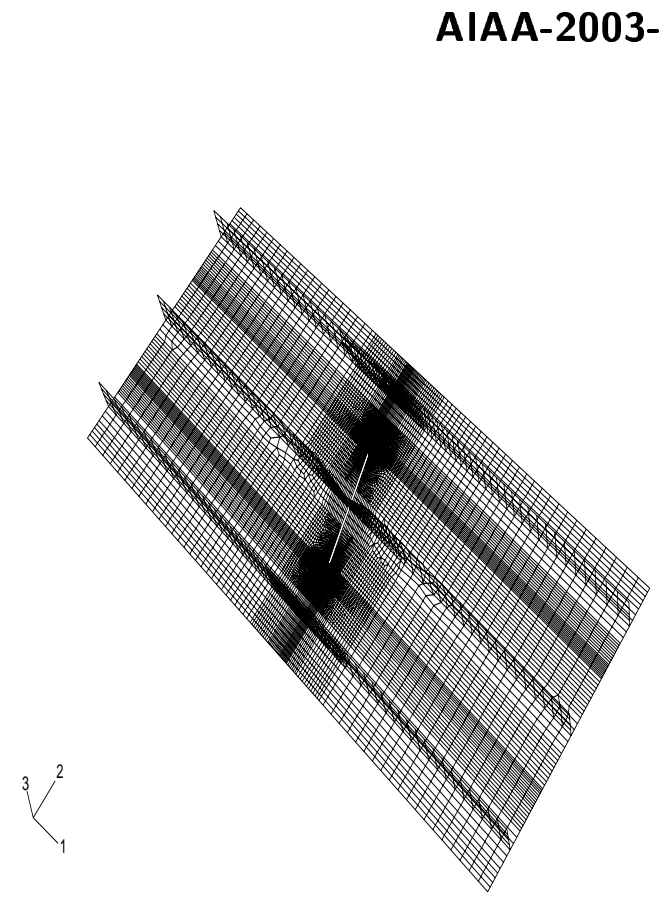

Figure 5: Finite element mesh of the panel. Close up view of the area ahead of the notch tip follows in the next figure.

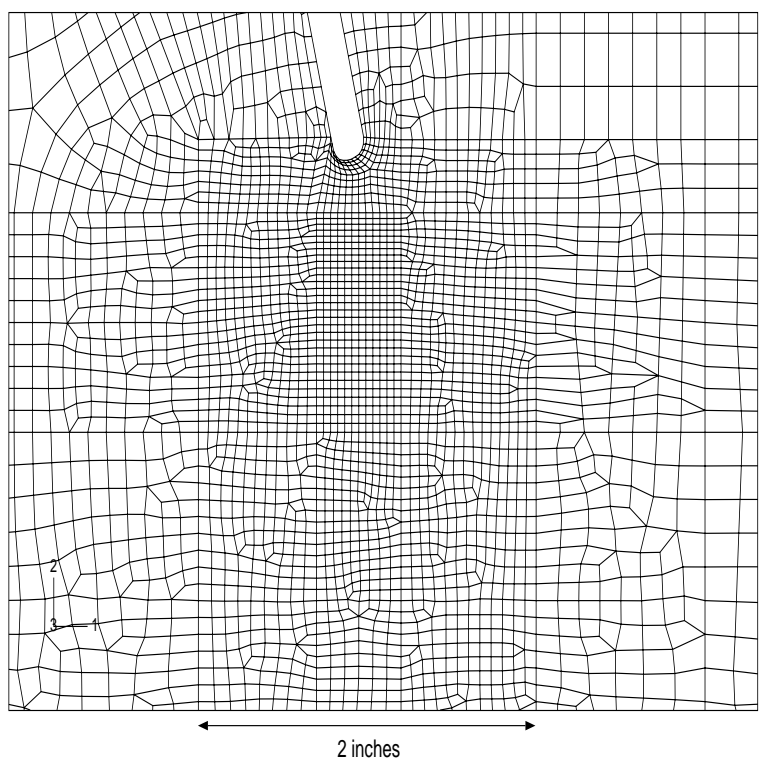

Continuation of Figure 5 
AIAA-2003-1598

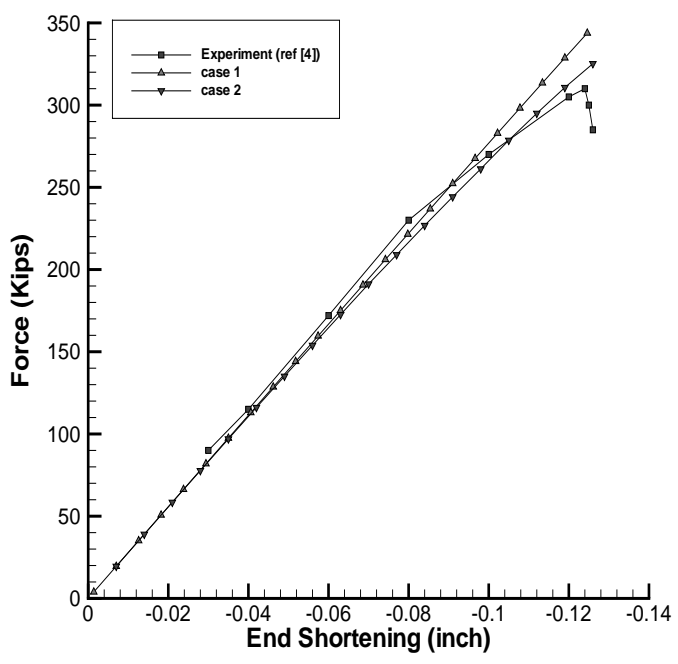

Figure 6: Load-Displacement response of the panel

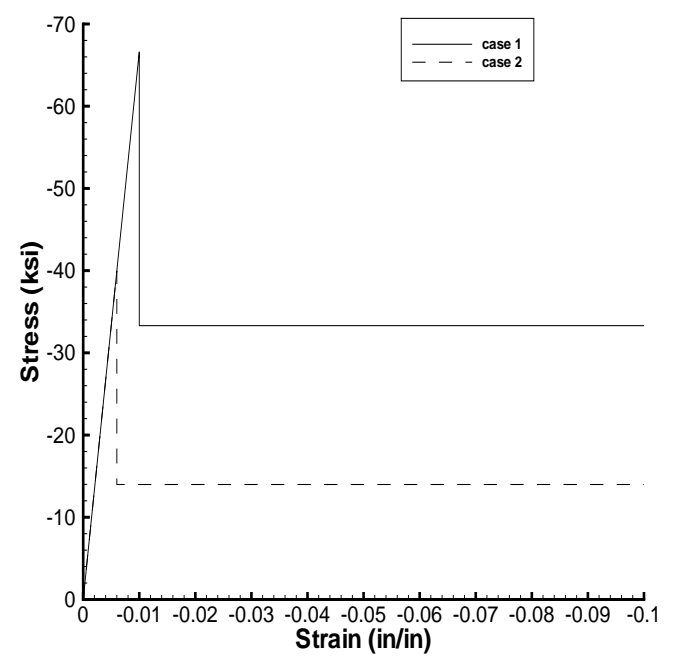

Figure 7: Stress-strain curves input to ABAQUS (axial direction)

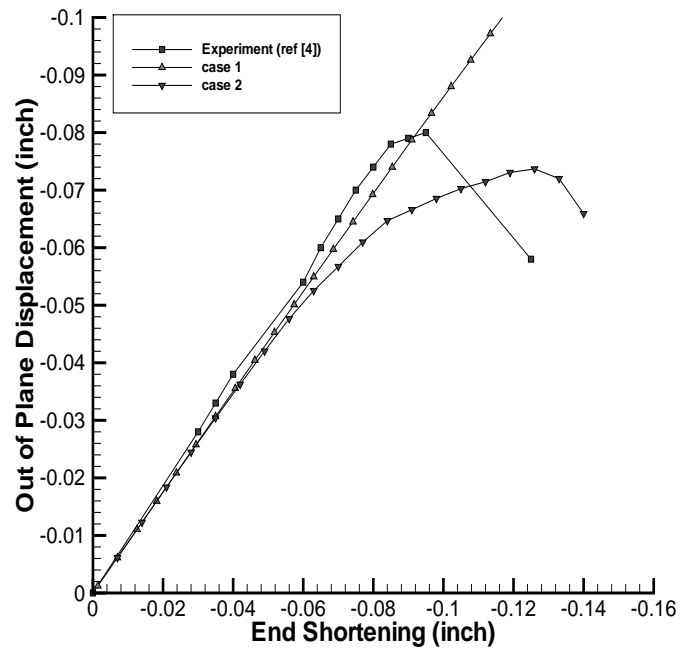

Figure 8: Out of Plane-Displacement response of the panel

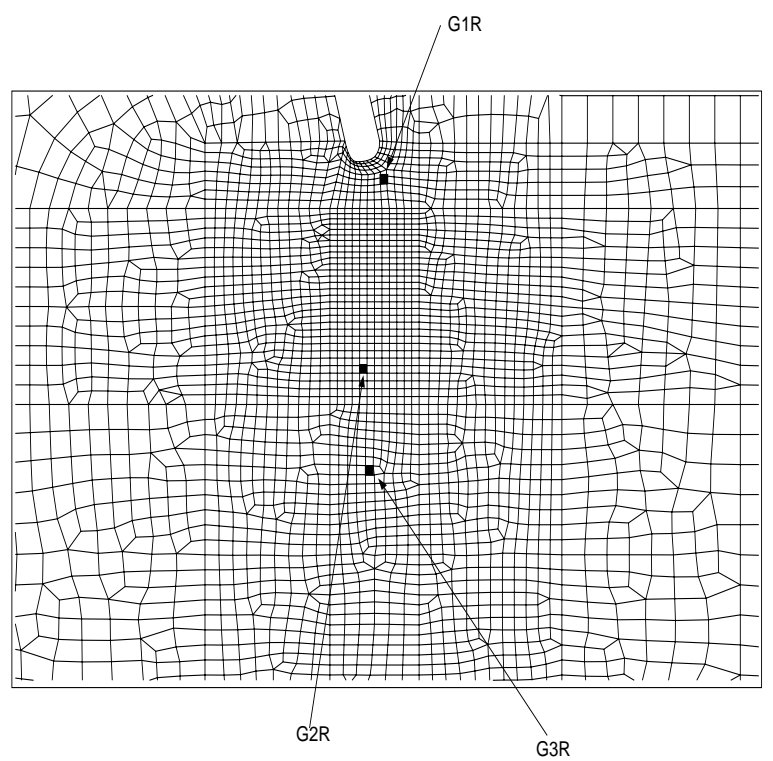

Figure 9: Locations for strain measurement (gages G1R, G2R, G3R, [4])

10

American Institute of Aeronautics and Astronautics 
AIAA-2003-1598

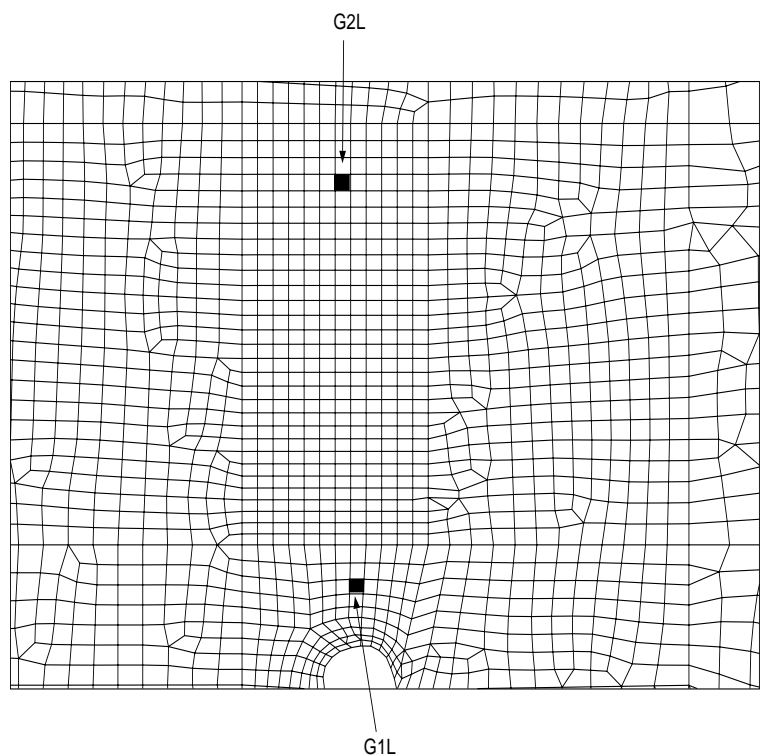

Figure 10: continuation of Figure 9 (gages G1L, G2L [4])

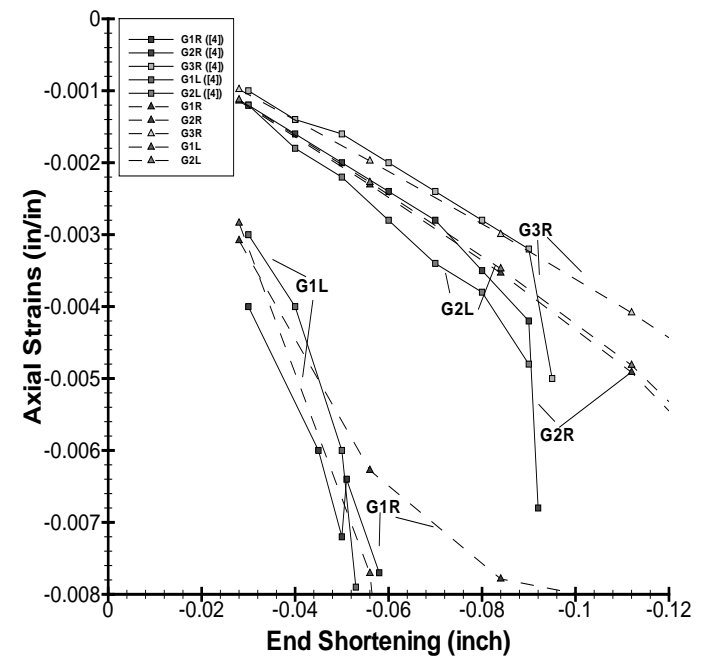

Figure 11: Results from P-15, strains at 5 locations (EST, NASA) 